\title{
DISTRIBUIÇÃO ESPACIAL E ASPECTOS EPIDEMIOLÓGICOS DA LEISHMANIOSE TEGUMENTAR AMERICANA NO MUNICÍPIO DE IMBÉ DE MINAS, LESTE DO ESTADO DE MINAS GERAIS, BRASIL
}

\section{SPATIAL DISTRIBUTION AND EPIDEMIOLOGICAL ASPECTS OF AMERICAN TEGUMENTARY LEISHMANIOSIS IN THE MUNICIPALITY OF IMBÉ DE MINAS, EAST OF THE STATE OF MINAS GERAIS, BRAZIL}

Rafael Luiz da Silva Neves
Doutorando em Ciências da Saúde
Instituto René Rachou - Fiocruz Minas
rafaelluizneves@hotmail.com
Andreza do Nascimento Gonçalves
Enfermeira da Secretaria Municipal de Saúde de Imbé de Minas
andrezanascimento@ @hotmail.com
Daniel Rodrigues Silva
Doutor em Farmácia
Centro Universitário de Caratinga
danfarma@bol.com.br
Juscélio Clemente de Abreu
Doutor em Genética
Centro Universitário de Caratinga
biotec.abreu@gmail.com
Gabriel Menezes Wernersbach
Acadêmico de Medicina Veterinária
Universidade Federal de Viçosa
gabriel.wernersbach@ufv.br
Carlos Henrique Wernersbach Guerra
Doutor em Geografia
Centro Universitário de Caratinga
carloshwguerra@gmail.com

\section{RESUMO}

A Leishmaniose Tegumentar Americana (LTA) é causada por protozoários pertencentes ao gênero Leishmania e representa um importante problema de saúde pública. O objetivo deste estudo foi analisar a distribuição espacial e caracterizar o perfil epidemiológico da LTA no município de Imbé de Minas (MG) utilizando como metodologia ferramentas de análise espacial e informações obtidas nos dados coletados no Serviço de Epidemiologia do município. Os resultados apresentados demonstraram que a incidência de LTA no município de Imbé de Minas vem aumentando a partir do ano 2015 e dobrando em 2018, sendo a maior parte dos acometidos, homens, residentes na zona rural, com baixa escolaridade e com idade entre 30 e 59 anos. Os 46 casos registrados apresentavam lesões cutâneas, diagnosticados em sua maioria por exame parasitológico direto. A droga mais utilizada no tratamento foi o Antimonial pentavalente. A quase totalidade dos casos encontra-se nas regiões norte e noroeste do município. Sugere-se às autoridades municipais a adoção de medidas preventivas dirigidas à população como uso de repelentes e manejo ambiental, medidas de controle de vetores, reservatórios e atividades de educação em saúde, além da melhoria do fluxo de notificação da LTA no município.

Palavras-chave: Leishmaniose Tegumentar Americana. Epidemiologia. Análise espacial.

Recebido em: 19/02/2020

Aceito para publicação em: 24/08/2020. 
Distribuição espacial e aspectos epidemiológicos da leishmaniose tegumentar americana no município de Imbé de Minas, leste do estado de Minas Gerais, Brasil
Rafael Luiz da Silva Neves Andreza do Nascimento Gonçalves Daniel Rodrigues Silva Juscélio Clemente de Abreu Gabriel Menezes Wernersbach Carlos Henrique Wernersbach Guerra

\begin{abstract}
American Tegumentary Leishmaniasis (ATL) is caused by protozoa belonging to the Leishmania genus and represents an important public health problem. The aim of this study was to analyze the spatial distribution and to characterize the epidemiological profile of ATL in the municipality of Imbé de Minas using spatial analysis tools and information obtained from data collected at the Municipal Epidemiology Service as a methodology. The results presented showed that the incidence of ATL in the municipality of Imbé de Minas has been increasing since 2015 and doubling in 2018, with the majority of those affected, men, living in the rural area, with low education and aged between 30 and 59 years. The 46 registered cases had cutaneous lesions, mostly diagnosed by direct parasitological examination. The drug most used in the treatment was Pentavalent Antimonial. Almost all cases are found in the north and northwest regions of the municipality. It is suggested to the municipal authorities to adopt preventive measures aimed at the population, such as the use of repellents and environmental management, vector control measures, reservoirs and health education activities, in addition to improving the flow of ATL notification in the municipality.
\end{abstract}

Keywords: American Tegumentary Leishmaniasis. Epidemiology. Spatial analysis.

\title{
INTRODUÇÃO
}

As Leishmanioses são doenças infecto-parasitárias que acometem o homem e são causadas por várias espécies de protozoários pertencentes ao gênero Leishmania (ROSS, 1903). Tais doenças representam um importante problema de saúde pública em vários países do mundo e são transmitidas aos seres humanos e outros mamíferos pela picada de flebotomíneos fêmeas (Diptera: Psychodidae), pertencentes aos gêneros Lutzomyia (França, 1924) e Phlebotomus (Rondani, 1840), infectadas durante o repasto sanguíneo em hospedeiros vertebrados (LAINSON, SHAW, 1978; DESJEUX, 2004). Fatores relacionados com o clima, o econômico, o ambiental, e o social, influenciam o ciclo de transmissão das leishmanioses e, assim, determinam os padrões epidemiológicos da doença (REITHINGER et al., 2007; CÁRDENAS et al., 2008).

Nos mamíferos, tais como, cães, marsupiais, roedores e humanos, os parasitos do gênero Leishmania vivem e se multiplicam intracelularmente em células fagocíticas. Embora tais espécies sejam morfologicamente muito semelhantes, elas causam diferentes formas clínicas: cutânea, mucosa, disseminada e difusa, também classificadas de formas dérmicas ou Leishmaniose Tegumentar (LT) e a forma visceral, Leishmaniose Visceral (LV), forma mais grave da doença. (SHAW et al., 2015; AKHOUNDI et al., 2016; ESPINOSA et al., 2016).

Globalmente, a LT é amplamente distribuída. Nas Américas, os casos de Leishmaniose Tegumentar Americana (LTA) são notificados em uma ampla área geográfica que se estende do sul dos Estados Unidos ao norte da Argentina (ALVAR et al., 2012). Nas últimas décadas, tornou-se evidente que a LTA é mais prevalente em áreas da América Latina do que se pensava anteriormente. Essa incerteza sobre sua ocorrência se deve, em parte a sua subnotificação e a limitação das informações coletadas (YADÓN et al, 2001).

Historicamente, a LTA tem sido caracterizada no Brasil como uma zoonose, endêmica principalmente nas áreas rurais, afetando mamíferos, e, ocasionalmente, homens, especialmente quando estes são expostos ao ciclo de transmissão silvestre da doença. Tal situação ocorreu frequentemente durante a construção de rodovias ou ferrovias (PASSOS et al., 1993). Durante a década de 1980, o Brasil teve um aumento de casos LTA e hoje, as infecções por esta doença e sua transmissão ativa são relatadas em regiões quase ou completamente desmatadas e em áreas periurbanas e urbanas (SOARES et al., 2014).

Em Minas Gerais, estudos chamaram a atenção, principalmente para áreas com altas taxas de incidência de LTA no Vale do Jequitinhonha (GONTIJO et al., 2002; BARATA et al., 2011), norte do estado (QUARESMA et al., 2011) e casos autóctones de LTA vêm sendo notificados em diversas cidades de Minas Gerais (CARDOSO et al., 2019). 
Distribuição espacial e aspectos epidemiológicos da leishmaniose tegumentar americana no município de Imbé de Minas, leste do estado de Minas Gerais, Brasil
Rafael Luiz da Silva Neves Andreza do Nascimento Gonçalves Daniel Rodrigues Silva Juscélio Clemente de Abreu Gabriel Menezes Wernersbach Carlos Henrique Wernersbach Guerra

Para supervisionar e controlar a LTA, foi lançado pelo Ministério da Saúde um programa de vigilância, com um objetivo de identificar e monitorar unidades territoriais de importância epidemiológica e desenvolver medidas de controle. Nesse sentido, as análises epidemiológicas são ferramentas poderosas para coletar informações sobre a epidemiologia das doenças infecciosas (BRASIL, 2017).

Uma compreensão abrangente da transmissão da LTA, incorporando o conhecimento da distribuição espacial, temporal e outras ferramentas epidemiológicas, específicas da doença, são essenciais para a tomada de decisões, a fim de direcionar e priorizar ações de estratégias de vigilância e controle (KAWA et al., 2010). Neste contexto, estudos de transmissão em áreas endêmicas são necessários, pois a falta de conhecimentos ecoepidemiológicos pode contribuir para o aumento da transmissão e manutenção da LTA em áreas endêmicas (GONTIJO, MELO, 2004).

O presente estudo deverá contribuir para o avanço do conhecimento sobre a LTA e caracterizar seu perfil epidemiológico no município de Imbé de Minas, Minas Gerais, Brasil, no intuito de fornecer informações que permitam ao poder público e à comunidade adotar medidas eficazes de combate à doença.

\section{MATERIAL E MÉTODOS}

A pesquisa foi realizada no município de Imbé de Minas, localizado no Leste Mineiro (19 $35^{\prime} 44^{\prime \prime} \mathrm{S}$ e 41 ${ }^{\circ} 58^{\prime} 09^{\prime \prime}$ ), Brasil. Possui área de aproximadamente $196,735 \mathrm{Km}^{2}$ e população estimada em 6.865 pessoas no ano de 2018, segundo o Instituto Brasileiro de Geografia e Estatística (IBGE, 2018).

Trata-se de um estudo longitudinal e retrospectivo, que utilizou dados oriundos das Fichas de Investigação de Leishmaniose Tegumentar Americana do Sistema de Informação de Agravos de Notificação - SINAN, fornecidas pelo Serviço de Epidemiologia da Secretaria de Saúde do município de Imbé de Minas.

Neste estudo, foram utilizadas informações referentes aos casos novos ocorridos no período de janeiro de 2013 a julho de 2018, espacializados por local de residência. Dentre as informações, foram utilizadas: sexo, faixa etária, escolaridade, ocupação, evolução e procedência dos casos, zona de moradia, tipo de lesão, exames laboratoriais realizados para o diagnóstico e tipo de tratamento.

Posteriormente, foram realizadas visitas in loco, para coleta de coordenadas geográficas, avaliação dos peridomicílios e da distância entre as casas dos infectados e o ambiente favorável à proliferação do vetor.

O aparelho de GPS utilizado para coleta dos dados foi o Garmin 76 , e as técnicas utilizadas para elaboração de mapas foram: mapeamento por estimativa de densidade de Kernel, e cálculo de Centro Médio.

Todas as informações foram tabuladas utilizando o software Excel 2013. As análises estatísticas foram feitas com o software R Estatística (versão 3.5.3) e os mapas foram gerados utilizando o ArcGIS Desktop 10.5 do Laboratório de Cartografia de Centro Universitário de Caratinga. Para utilização do Estimador de Densidade de Kernel, adotou-se células com tamanho 0.00065624358 tanto para $x$ quanto $y$, e não houve ponderação pela distribuição populacional, utilizando-se exclusivamente os casos registrados.

Para o teste de Regressão Linear (ano $x n^{\circ}$ de casos) utilizou-se os dados segundo o semestre de ocorrência para aumentar a significância estatística da análise.

\section{RESULTADOS E DISCUSSÃO}

Após levantamento nos registros do Serviço de Epidemiologia do município, foram encontradas 46 notificações de casos de Leishmaniose Tegumentar Americana no período analisado. Os dados sociodemográficos estão expostos na Tabela 1 e os dados referentes às notificações na Tabela 2.

Durante o período estudado, o município de Imbé de Minas notificou 46 casos de LTA, sendo que em nenhum dos casos houve lesões mucosas. Os casos apresentam uma linha de tendência ascendente (Gráfico 1). 
Distribuição espacial e aspectos epidemiológicos da leishmaniose tegumentar americana no município de Imbé de Minas, leste do estado de Minas Gerais, Brasil
Rafael Luiz da Silva Neves Andreza do Nascimento Gonçalves Daniel Rodrigues Silva Juscélio Clemente de Abreu Gabriel Menezes Wernersbach Carlos Henrique Wernersbach Guerra

Tabela 1 - Dados sociodemográficos dos casos de Leishmaniose Tegumentar Americana do município de Imbé de Minas - MG, notificados de janeiro de 2013 a julho de 2018

\begin{tabular}{|c|c|c|c|}
\hline Variável & Número & $\%$ & $\begin{array}{c}\text { Incidência } \\
\text { (casos novos } \\
100.000 \text { hab) }\end{array}$ \\
\hline \multicolumn{4}{|l|}{ Sexo } \\
\hline Masculino & 24 & 52,0 & 683,6 \\
\hline Feminino & 22 & 48,0 & 665,3 \\
\hline \multicolumn{4}{|l|}{ Faixa Etária } \\
\hline 0 a 14 anos & 10 & 21,7 & 606,8 \\
\hline 15 a 29 anos & 7 & 15,2 & 443,0 \\
\hline 30 a 59 anos & 22 & 47,8 & 803,8 \\
\hline 60 anos e mais & 7 & 15,2 & 820,6 \\
\hline \multicolumn{4}{|l|}{ Escolaridade } \\
\hline Analfabeto & 2 & 4,3 & - \\
\hline $1^{a}$ a $4^{a}$ série incompleta & 19 & 41,3 & - \\
\hline $4^{a}$ série completa & 8 & 17,4 & - \\
\hline $5^{\mathrm{a}}$ a $8^{\mathrm{a}}$ série incompleta & 2 & 4,3 & - \\
\hline Ensino Fundamental completo & 3 & 6,5 & - \\
\hline Ensino Médio incompleto & 1 & 2,2 & - \\
\hline Ensino Médio completo & 2 & 4,3 & - \\
\hline Ignorado & 6 & 13,0 & - \\
\hline Não se aplica & 3 & 6,5 & - \\
\hline \multicolumn{4}{|l|}{ Ocupação } \\
\hline Gerente de prod. e op. agrícola & 2 & 4,3 & - \\
\hline Doméstica & 5 & 10,9 & - \\
\hline $\begin{array}{l}\text { Trabalhador volante da } \\
\text { agricultura }\end{array}$ & 6 & 13,0 & - \\
\hline Estudante & 8 & 17,4 & - \\
\hline Lavrador & 10 & 21,7 & - \\
\hline Dona de casa & 5 & 10,9 & - \\
\hline Aposentado & 6 & 13,0 & - \\
\hline Ignorado & 4 & 8,7 & - \\
\hline
\end{tabular}

Fonte: Fichas de notificação de Leishmaniose Tegumentar Americana, Serviço de Epidemiologia, Imbé de Minas - Minas Gerais. 
Distribuição espacial e aspectos epidemiológicos da leishmaniose tegumentar americana no município de Imbé de Minas, leste do estado de Minas Gerais, Brasil
Rafael Luiz da Silva Neves Andreza do Nascimento Gonçalves Daniel Rodrigues Silva Juscélio Clemente de Abreu Gabriel Menezes Wernersbach Carlos Henrique Wernersbach Guerra

Tabela 2 - Dados referentes aos casos de Leishmaniose Tegumentar Americana do município de Imbé de Minas - MG, notificados de janeiro de 2013 a julho de 2018

\begin{tabular}{|c|c|c|c|}
\hline & & & Incidência (casos \\
\hline Variável & Número & $\%$ & $\begin{array}{l}\text { novos/ } 100.000 \\
\text { hab) }\end{array}$ \\
\hline Zona de ocorrência & & & \\
\hline Zona urbana & 2 & 4,3 & - \\
\hline Zona rural & 44 & 95,7 & - \\
\hline Local de ocorrência & & & \\
\hline Córrego dos Miguel & 10 & 21,7 & - \\
\hline Córrego do Ouro & 8 & 17,4 & - \\
\hline Córrego da Palhadinha & 6 & 13,0 & - \\
\hline Córrego dos Campos & 4 & 8,7 & - \\
\hline Demais localidades & 18 & 39,1 & - \\
\hline Ano do diagnóstico & & & \\
\hline 2018 (até julho) & 14 & 30,4 & 203,93 \\
\hline 2017 & 16 & 34,8 & 231,92 \\
\hline 2016 & 7 & 15,2 & 102,01 \\
\hline 2015 & 4 & 8,7 & 58,36 \\
\hline 2014 & 3 & 6,5 & 44,23 \\
\hline 2013 & 2 & 4,3 & 29,69 \\
\hline Mês do diagnóstico & & & \\
\hline Dezembro & 2 & 4,3 & 29,3 \\
\hline Novembro & 2 & 4,3 & 29,3 \\
\hline Outubro & $\overline{1}$ & 2,2 & 14,6 \\
\hline Setembro & 8 & 17,4 & 117,2 \\
\hline Agosto & 4 & 8,7 & 58,6 \\
\hline Julho & 2 & 4,3 & 29,3 \\
\hline Junho & 2 & 4,3 & 29,3 \\
\hline Maio & 6 & 13,0 & 87,9 \\
\hline Abril & 3 & 6,5 & 43,9 \\
\hline Março & 6 & 13,0 & 87,9 \\
\hline Fevereiro & 3 & 6,5 & 43,9 \\
\hline Janeiro & 7 & 15,2 & 102,5 \\
\hline Critério de confirmação & & & \\
\hline Teste laboratorial & 40 & 87,0 & - \\
\hline Clínico-epidemiológico & 6 & 13,0 & - \\
\hline Teste Intradermorreação & & & \\
\hline Realizadas & 16 & 34,8 & - \\
\hline Positivas & 12 & 75,0 & - \\
\hline Exame parasitológico dir & & & \\
\hline Realizados & 39 & 84,8 & - \\
\hline Positivos & 37 & 94,9 & - \\
\hline Presença de lesão por LT & & & \\
\hline Cutânea & 46 & 100,0 & - \\
\hline Mucosa & 0 & 0,0 & - \\
\hline Droga utilizada no tratam & & & \\
\hline Antimonial pentavalente & 31 & 67,4 & - \\
\hline Anfotericina B & 3 & 6,5 & - \\
\hline Outras drogas & 11 & 23,9 & - \\
\hline Nenhuma droga & 1 & 2,2 & - \\
\hline Evolução do caso & & & \\
\hline Cura & 36 & 78,3 & - \\
\hline Abandono & 2 & 4,3 & - \\
\hline Óbito por outras causas & 2 & 4,3 & - \\
\hline Transferência & 1 & 2,2 & - \\
\hline Continuam em tratamento & 5 & 10,9 & - \\
\hline
\end{tabular}

Fonte: Fichas de notificação de Leishmaniose Tegumentar Americana, Serviço de Epidemiologia, Imbé de Minas - Minas Gerais. 
Distribuição espacial e aspectos epidemiológicos da leishmaniose tegumentar americana no município de Imbé de Minas, leste do estado de Minas Gerais, Brasil
Rafael Luiz da Silva Neves Andreza do Nascimento Gonçalves Daniel Rodrigues Silva Juscélio Clemente de Abreu Gabriel Menezes Wernersbach Carlos Henrique Wernersbach Guerra

Gráfico 1 - Teste de Regressão Linear Simples dos casos de Leishmaniose diagnosticados no município de Imbé de Minas - MG, entre janeiro de 2013 a julho de 2018

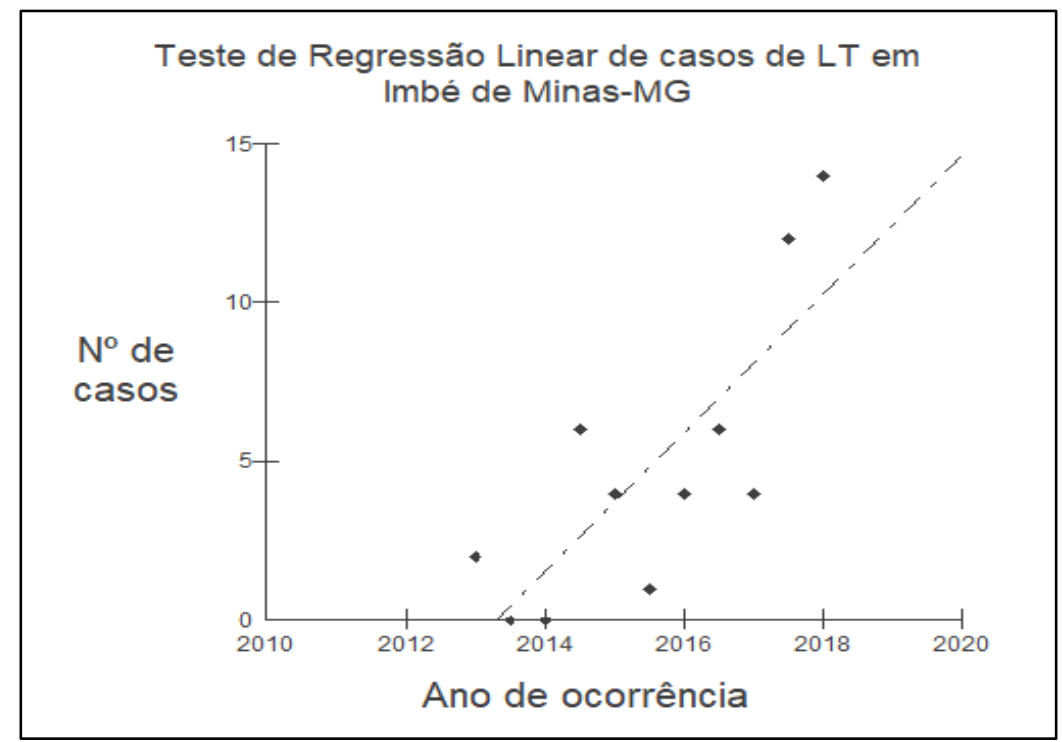

Coef. de Regressão: 2,1818; $p=0,0038$;

Fonte: Dados coletados pelos autores no Serviço de Epidemiologia, Imbé de Minas - Minas Gerais.

Segundo os critérios de classificação epidemiológica da Leishmaniose Tegumentar Americana (LTA) o município de Imbé de Minas é classificado como "Município com transmissão", definido como "municípios com histórico de registro de casos humanos autóctones de Leishmaniose Tegumentar (LT), contínuos ou não, nos últimos três anos", onde está havendo "Ocorrência de surto", definido como "presença de casos de LT em uma área sem transmissão/silenciosa ou aumento de casos em relação ao número esperado em áreas com transmissão". (BRASIL, 2017).

A incidência de LTA aumentou 7,81 vezes entre o ano de 2013 (29,69/100.000 habitantes) e o ano 2017 (231,92/100.000 habitantes). A Incidência média foi igual a 111,74/100.000 habitantes, e seu desvio padrão igual a 86,19. A densidade de casos por área (n. ${ }^{\circ}$ de casos de LT por $\mathrm{Km}^{2}$ ) nos últimos analisados três anos, conforme foi de 0,18 casos $/ \mathrm{Km}^{2}$.

Considerando que os dados de 2018 referem-se aos meses de janeiro a julho, espera-se que a taxa de incidência neste ano supere a do ano 2017. A Figura 1 apresenta a distribuição espacial dos casos de LTA, segundo o ano de diagnóstico.

Em relação às pessoas diagnosticadas, observa-se que não houve predominância significativa do sexo masculino (52,0\% com taxa de incidência de 683,6 casos $/ 100.000$ habitantes) em relação ao feminino (48,0\% com taxa de incidência de 665,3 casos/100.000 habitantes) como aponta a literatura (BRASIL, 2009; COSTA et al., 2018).

De acordo com Oliart-Guzmán et al., (2013) e Fonseca et al., (2014) o percentual de homens acometidos pela LTA é maior, pela exposição frequente ao ambiente extradomiciliar devido a atividades no meio rural, em locais próximos a matas, o principal habitat do inseto vetor das Leishmanioses. No entanto, vale ressaltar também que, embora a transmissão esteja associada às atividades masculinas, Eid et al., (2018) sugerem que a incidência em mulheres e crianças pode estar relacionada à observação de que toda a família vai para o local de trabalho, principalmente com bebês mantidos à sombra ou abrigos temporários montados em áreas de cultivo; além disso, alguns homens têm o potencial de uma maior suscetibilidade clínica (DAVIES et al., 1997; COSTA et al., 1998; LOCKARD et al., 2019), embora em cenários de alta exposição esse viés de gênero tenda a diminuir (FONSECA et al., 2014; SOARES et al., 2014). 
Distribuição espacial e aspectos epidemiológicos da leishmaniose tegumentar americana no município de Imbé de Minas, leste do estado de Minas Gerais, Brasil
Rafael Luiz da Silva Neves Andreza do Nascimento Gonçalves Daniel Rodrigues Silva Juscélio Clemente de Abreu Gabriel Menezes Wernersbach Carlos Henrique Wernersbach Guerra

Figura 1 - Distribuição espacial dos casos de LTA em Imbé de Minas - MG, segundo o ano do diagnóstico

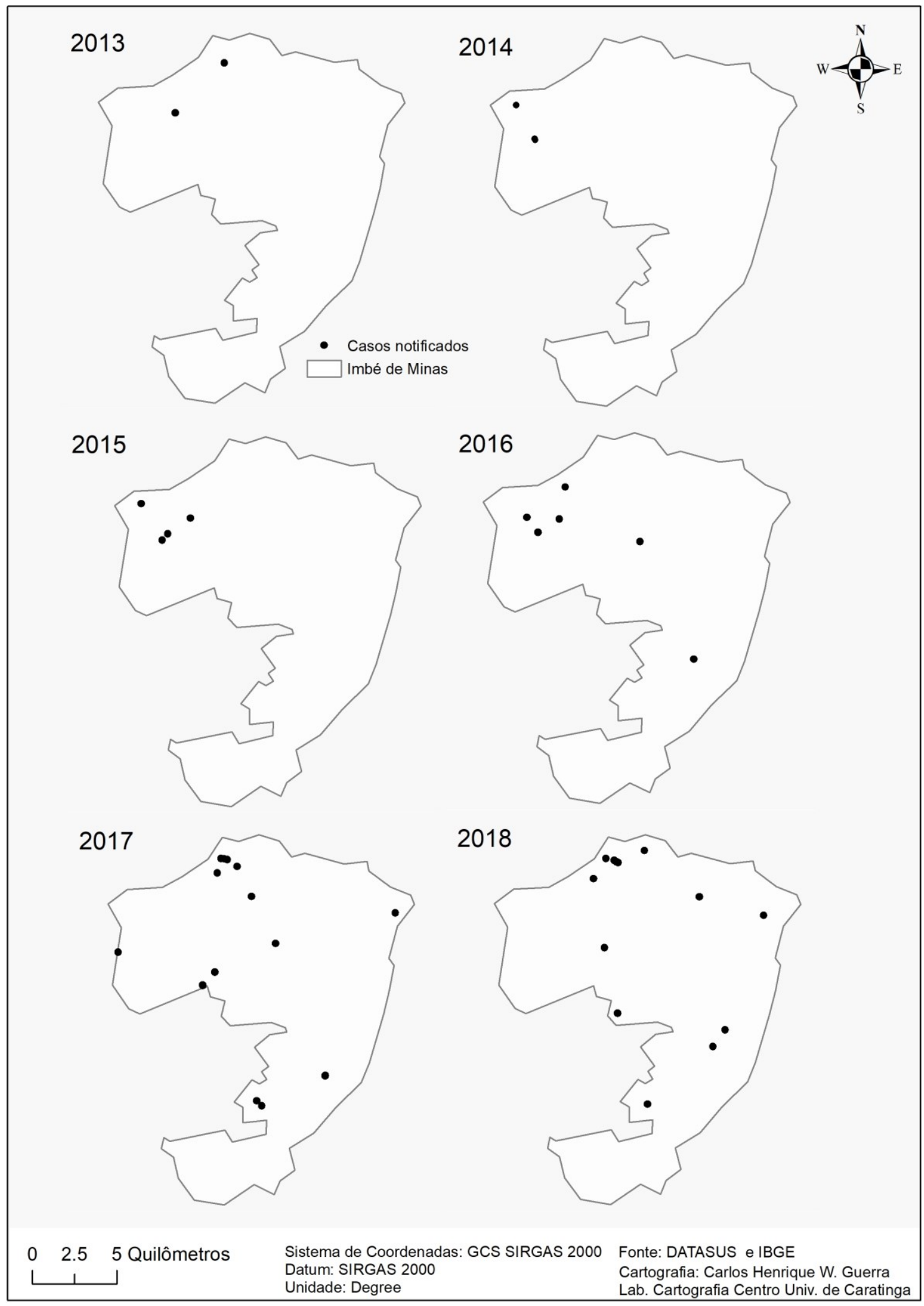

Fonte: Dados coletados pelos autores no Serviço de Epidemiologia, Imbé de Minas - Minas Gerais 
Distribuição espacial e aspectos epidemiológicos da leishmaniose tegumentar americana no município de Imbé de Minas, leste do estado de Minas Gerais, Brasil
Rafael Luiz da Silva Neves Andreza do Nascimento Gonçalves Daniel Rodrigues Silva Juscélio Clemente de Abreu Gabriel Menezes Wernersbach Carlos Henrique Wernersbach Guerra

Outro dado sugestivo de infecção intradomiciliar ou peridomiciliar é a faixa etária dos casos. Ressaltase o significativo percentual de menores de 14 anos infectados $(21,7 \%)$, com uma incidência de 606,8 casos por 100.000 crianças. A infecção está predominante concentrada entre 30 e $59(47,8 \%)$ com incidência de 803,8 casos por 100.000 habitantes, diferentemente do estudo de Costa (2018), realizado na Amazônia Legal, onde a maior concentração de casos se deu na faixa dos 18 aos 29 anos. Ressaltase também que, como os flebótomos têm hábitos crepusculares, existe a possibilidade de infecção intra ou peridomiciliar, vistos que nos horários crepusculares as pessoas encontram-se, na maioria das vezes, em seus domicílios (MELO et., 2017; PINTO et al., 2020).

Os casos de LTA foram mais frequentes entre os que apresentavam baixa ou nenhuma escolaridade (45,6\%), mas, segundo dados do Censo de 2010 (IBGE, 2018), existiam no município todo $45,3 \%$ de pessoas nas mesmas condições de escolaridade. Tais resultados são corroborados por KaragiannisVoules et al. (2013).

Já em relação à ocupação, 39,0\% dos indivíduos, tinham atividades ligadas a área rural, como gerente de produção agrícola, lavrador ou trabalhador volante da agricultura. Já as domésticas/donas de casa respondem por $21,8 \%$. Uma vez que $95,7 \%$ os infectados moram na zona rural, não existe disparidade entre local de residência e local de trabalho.

Quanto aos casos notificado, $87,0 \%$ foram confirmados por critério laboratorial e 13,0\% por vínculo clínico-epidemiológico. O teste de Intradermorreação de Montenegro foi aplicado em $34,8 \%$ dos casos, sendo que destes, $75,0 \%$ foram positivos. O exame parasitológico direto foi realizado em $84,9 \%$ dos casos, sendo o índice de positividade de 94,9\%.

A droga mais utilizada no tratamento dos casos foi o Antimonial Pentavalente $(67,4 \%)$, sendo essa a droga de primeira escolha indicada para o tratamento da Leishmaniose Tegumentar. Até a data da coleta dos dados, $10,9 \%$ dos casos continuavam em tratamento, $78,3 \%$ foram curados, havendo abandono em $4,3 \%$ dos tratamentos.

Embora a LTA tenha modificação em seu perfil epidemiológico nos últimos anos, passando de uma doença adquirida no contato com áreas silvestres para transmissão em áreas urbanas, não é o que se observa neste estudo, uma vez que $95,7 \%$ dos casos ocorreram em moradores da zona rural do município. Nas áreas do município, chamada Córrego dos Miguel, assim como no chamado Córrego do Ouro, é possível observar que grande parte das casas não possuía distância mínima de 200 ou 300 metros das matas, além de possuírem animais domésticos como cães, galinhas e porcos que são que são reservatórios de espécies do gênero Leishmania, fato corroborado por Margonari et al. (2013) e Menezes et al. (2015).

Existe uma significativa concentração de casos em três localidades chamadas Córrego dos Miguel $(21,7 \%)$, Córrego do Ouro $(17,4 \%)$ e Córrego da Palhadinha $(13,0 \%)$. Estas áreas encontram-se localizadas na porção norte/noroeste do município como se observa no Mapa 1.

Observa-se também que as casas na localidade do Córrego dos Miguel são muito próximas umas das outras, além de existir uma área alagada para onde é drenado todo o esgoto, que corre a céu aberto, o que resulta no aumento de matéria orgânica nesse lugar, e poderia atrair reservatórios silvestres e sinantrópicos.

Na região norte/noroeste existem muitas áreas remanescentes de Mata Atlântica, entremeadas com lavouras de café, formando um extenso corredor ecológico ligando as três localidades mencionadas, o que pode facilitar a circulação do flebotomíneo e de reservatórios da Leishmania.

Para verificar a existência de deslocamento dos casos de LTA dentro do território no decorrer do período estudado, utilizou-se a técnica de Centro Médio. O resultado desta análise mostrou que os casos de LTA, notificados nos anos de 2016, 2017 e 2018 (Mapa 2), se deslocaram no sentido oesteleste, na mesma faixa territorial. Não foram incluídos os anos 2013, 2014 e 2015 nesta análise devido à insuficiência de casos notificados para que fosse significativo o resultado. 
Distribuição espacial e aspectos epidemiológicos da leishmaniose tegumentar americana no município de Imbé de Minas, leste do estado de Minas Gerais, Brasil
Rafael Luiz da Silva Neves Andreza do Nascimento Gonçalves Daniel Rodrigues Silva Juscélio Clemente de Abreu

Gabriel Menezes Wernersbach Carlos Henrique Wernersbach Guerra

Mapa 1 - Distribuição espacial dos casos de LT no município de Imbé de Minas - MG.

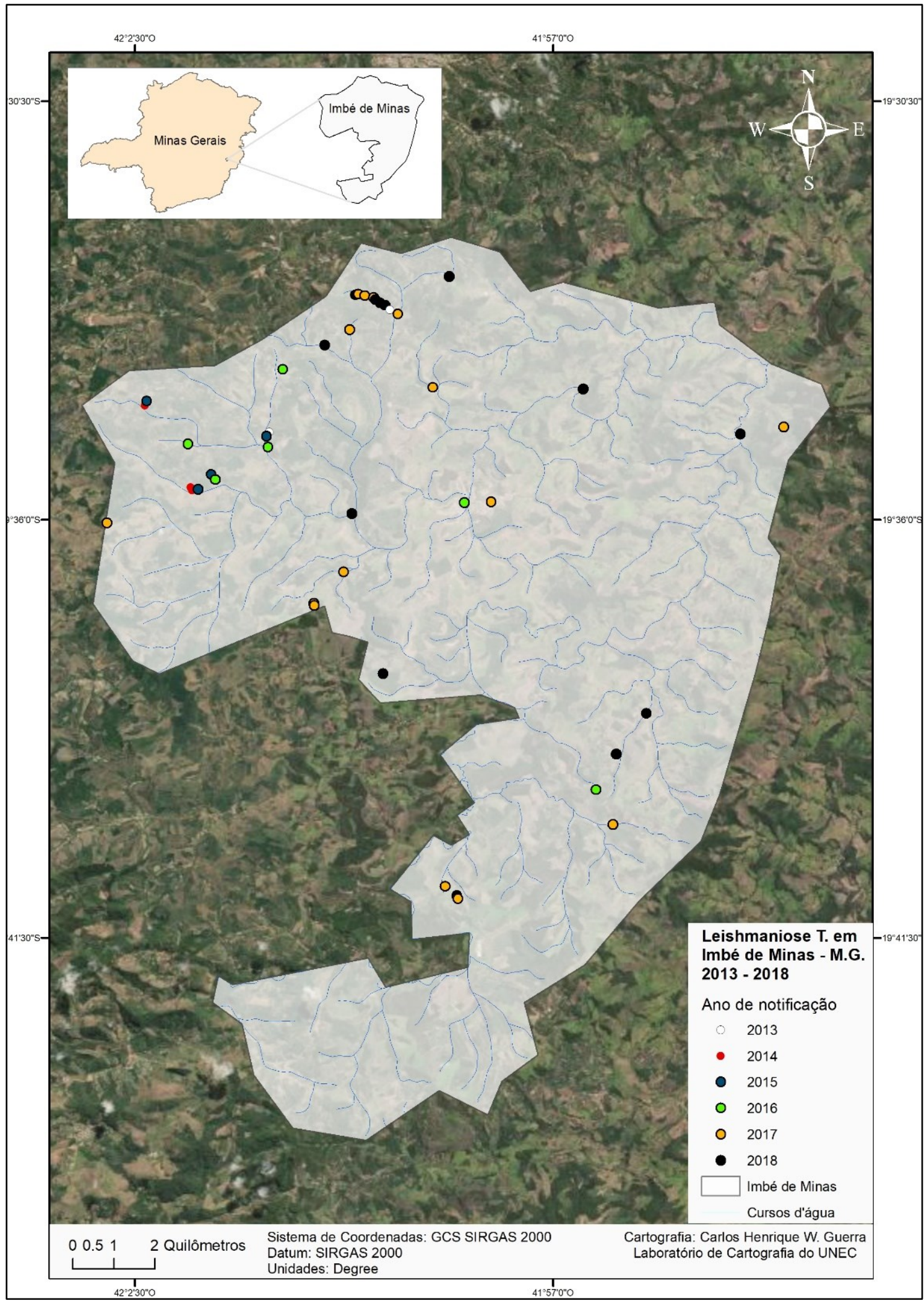

Fonte: Dados coletados pelos autores no Serviço de Epidemiologia, Imbé de Minas - Minas Gerais 
Distribuição espacial e aspectos epidemiológicos da leishmaniose tegumentar americana no município de Imbé de Minas, leste do estado de Minas Gerais, Brasil
Rafael Luiz da Silva Neves Andreza do Nascimento Gonçalves Daniel Rodrigues Silva Juscélio Clemente de Abreu Gabriel Menezes Wernersbach Carlos Henrique Wernersbach Guerra

Mapa 2 - Distribuição dos casos de LT, notificados em Imbé de Minas-MG entre 2013 e 2018, com destaque para os centros médios.

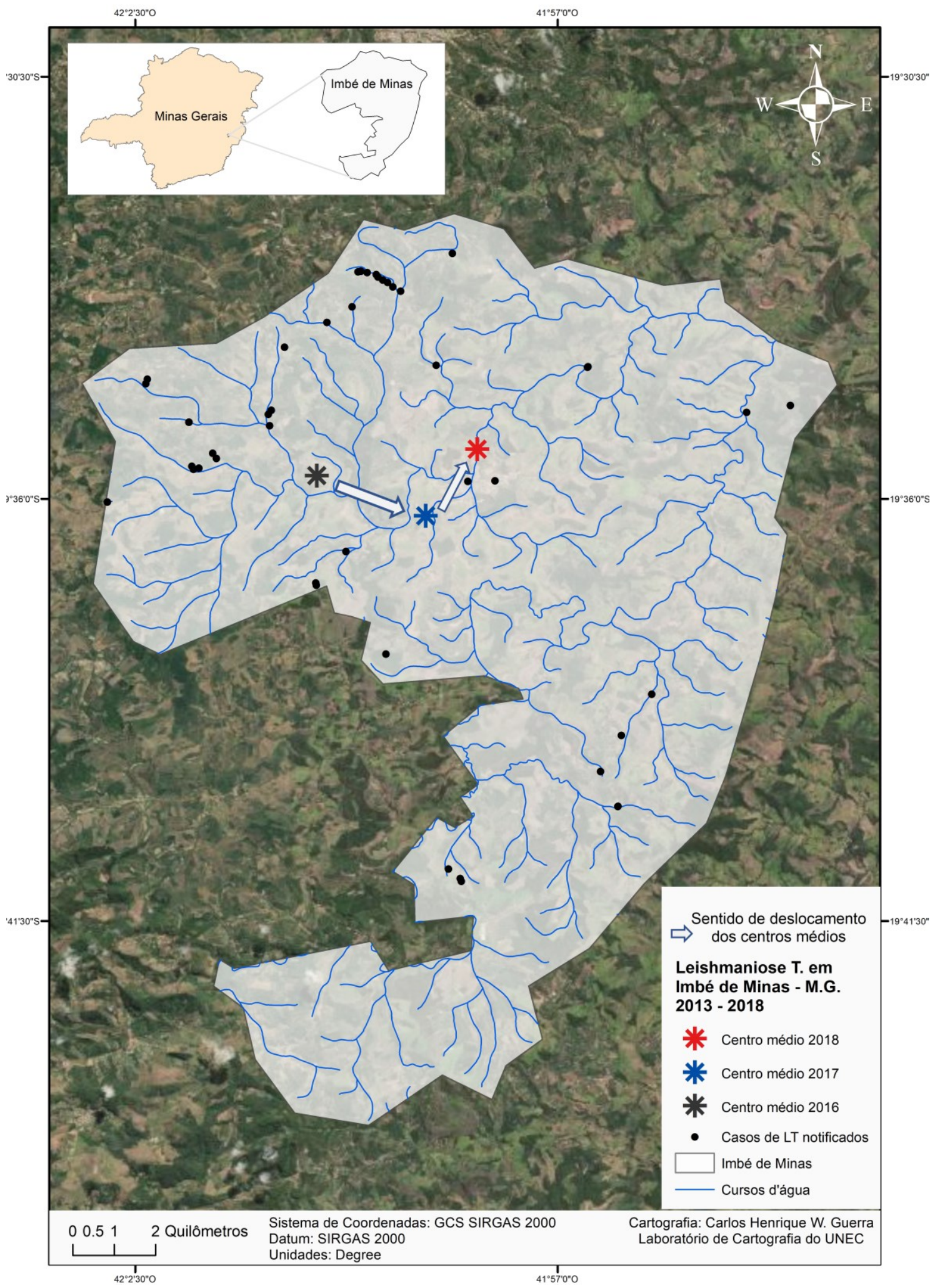

Fonte: Dados coletados pelos autores no Serviço de Epidemiologia, Imbé de Minas - Minas Gerais 
Distribuição espacial e aspectos epidemiológicos da leishmaniose tegumentar americana no município de Imbé de Minas, leste do estado de Minas Gerais, Brasil
Rafael Luiz da Silva Neves Andreza do Nascimento Gonçalves Daniel Rodrigues Silva Juscélio Clemente de Abreu

Gabriel Menezes Wernersbach Carlos Henrique Wernersbach Guerra

A análise de densidade de casos, apresentada no Mapa 3, aponta a maior concentração de casos no extremo norte do município, onde encontra-se localizado o Córrego dos Miguel, seguido da região noroeste, onde estão localizados o Córrego da Palhadinha, Córrego dos Amaros e Córrego das Palmeiras.

Mapa 3 - Densidade de Kernel dos casos de LT notificados no município de Imbé de Minas-MG entre 2013 e 2018.

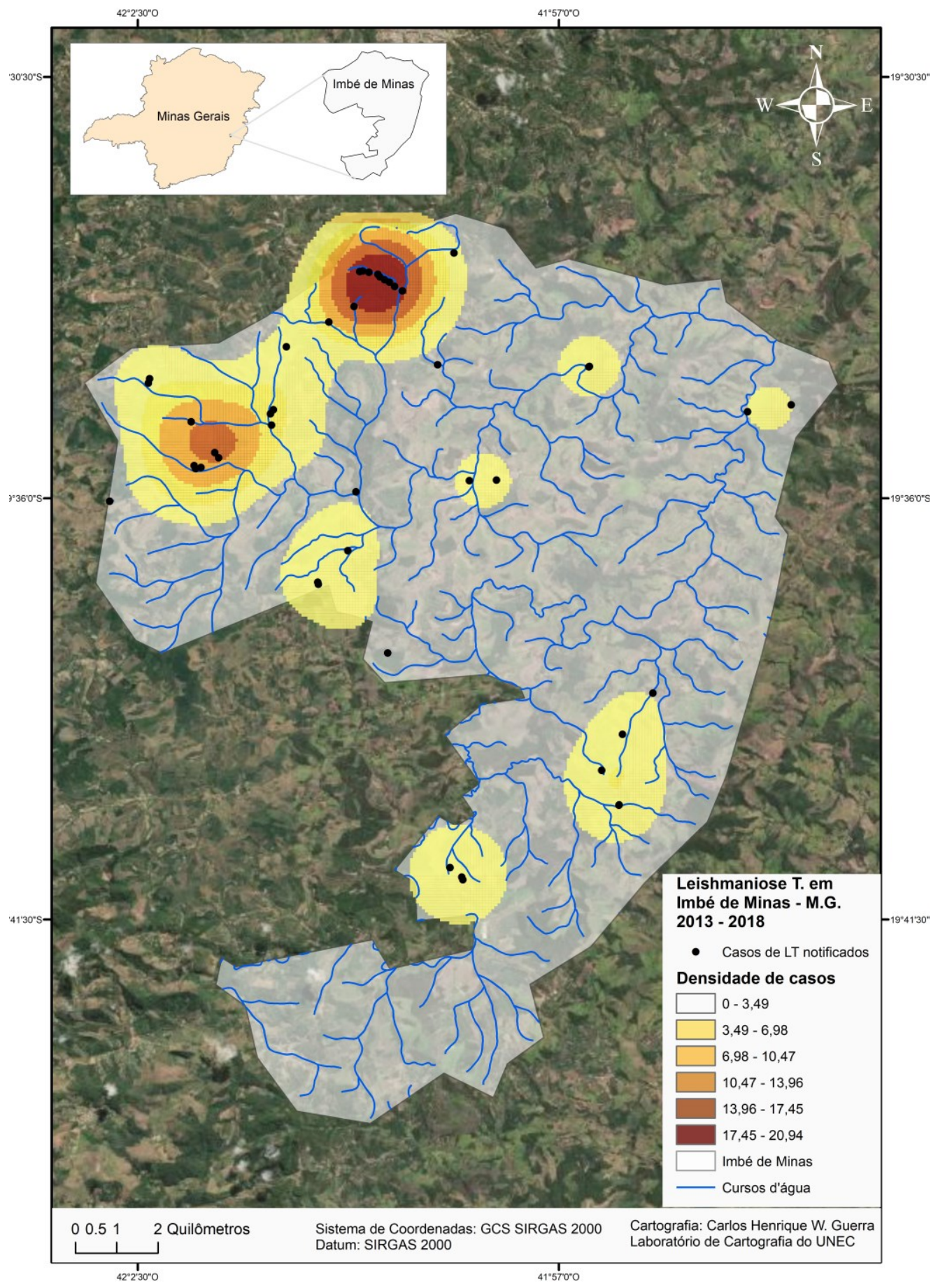

Fonte: Dados coletados pelos autores no Serviço de Epidemiologia, Imbé de Minas - Minas Gerais 
Distribuição espacial e aspectos epidemiológicos da leishmaniose tegumentar americana no município de Imbé de Minas, leste do estado de Minas Gerais, Brasil
Rafael Luiz da Silva Neves Andreza do Nascimento Gonçalves Daniel Rodrigues Silva Juscélio Clemente de Abreu Gabriel Menezes Wernersbach Carlos Henrique Wernersbach Guerra

Utilizando os dados da Estação Meteorológica Automática de Caratinga, distante 23,1 Km em linha reta do centro da cidade de Imbé de Minas, observa-se que a precipitação média mensal no período estudado varia entre $9,4 \mathrm{~mm}$ no mês de agosto e $1154,9 \mathrm{~mm}$ no mês de dezembro. O período mais seco do ano ocorre entre os meses de junho e setembro, coincidindo com as temperaturas médias mensais mais baixas (variando entre $18,9^{\circ} \mathrm{C}$ em junho e $21,9^{\circ} \mathrm{C}$ em setembro).

No período estudado, os meses com maior número de casos foram agosto e setembro, com sete casos cada, coincidindo com o período de menor precipitação média e menor temperatura média mensal. Destaca-se o fato de o número mensal de casos permanecer praticamente estável (variando de 2 a 4 ) no período de maior precipitação e temperaturas médias mais elevadas.

Com base nos dados analisados observa-se não existir associação estatisticamente significante entre o número de casos diagnosticados e a precipitação média mensal ( $r$-Pearson $=0,0260$ e $p=0,8596$ ) e o número de casos e a temperatura média mensal ( $r$-Pearson=0,0159 e $p=0,7982)$. Tal fato pode se justificar devido ao tempo de evolução da doença, e ou o momento em que a notificação e o diagnóstico do caso são feitos. Embora Guerra (2006), em estudo realizado em Manaus - AM, afirma que, nesta região, o número de casos aumenta no período chuvoso quando existe aumento da densidade de vetores. Mendes et al., (2016), afirma haver forte relação entre a transmissão da LTA e a precipitação, e o mesmo autor, citando Rey (2008), afirma que há indícios de que o período de maior transmissão ocorra durante ou logo após as estações chuvosas, em função do aumento da densidade populacional do inseto. Também não se observou correlação estatisticamente significativa. Fatores associados ao clima como temperatura, umidade e pluviosidade influenciam de modo variável a população de flebótomos, dependendo da região analisada. A sazonalidade dos flebotomíneos está relacionada com os padrões de distribuição de chuvas que agem modificando as condições dos criadouros no solo, sendo encontrados em altas densidades durantes os meses mais quentes e úmido, sendo ainda encontrados, em algumas áreas de exceção, durante os meses mais frios (NEGRÃO, 2009).

\section{CONSIDERAÇÕES FINAIS}

O presente estudo permitiu demonstrar que a incidência da LTA no município de Imbé de Minas - MG vem apresentando aumento acentuado a partir do ano 2015, mais do que dobrando em 2018, sendo os acometidos, em sua maioria homens, residentes na zona rural, com baixa escolaridade, com idade entre 30 e 59 anos, que trabalham como agricultores.

Em relação aos casos notificados, todos apresentavam apenas lesões cutâneas, diagnosticados em sua maioria por exame parasitológico direto (confirmação laboratorial), sendo a droga mais utilizada no tratamento o Antimonial pentavalente. A taxa de cura próxima a $78,3 \%$.

Quanto às características ambientais, não houve correlação entre a precipitação média e o número de casos diagnosticados. Embora sejam necessárias coletas entomológicas nas áreas com casos de LTA para auxiliar a corroborar esta informação. A quase totalidade dos casos encontram-se nas regiões Noroeste e Norte, com maior concentração em três córregos (dos Miguel, do Ouro e da Palhadinha), que totalizam $52,1 \%$ dos casos notificados. Detectou-se também que os casos de LTA vem se deslocando no sentido oeste-leste do município.

Sugere-se às autoridades municipais a adoção de medidas preventivas destinada à população como o uso de repelentes quando expostos a ambientes onde os vetores habitualmente possam ser encontrados, evitar a exposição nos horários de atividades do vetor, crepúsculo e noite, uso de mosquiteiros de malha fina, manejo ambiental por meio de limpeza de quintais e terrenos, destino adequando lixo orgânico e limpeza periódica dos abrigos de animais domésticos. Tais medidas preventivas devem ser combinadas com medidas de controle flexíveis, distintas e adequadas a cada região ou foco em particular considerando os aspectos epidemiológicos, bem como seus determinantes. Por isso se faz necessário a melhoria da notificação dos casos, evitando a subnotificação, para um melhor entendimento da epidemiologia da LTA.

\section{BIBLIOGRAFIA}

AKHOUNDI. M.; KUHLS, K.; CANNET, A.; VOTÝPKA, J.; MARTY, P.; DELAUNAY, P.; SERENO, D. A Historical Overview of the Classification, Evolution, and Dispersion of Leishmania Parasites and

DOI: http://dx.doi.org/10.14393/Hygeia16052808 $\quad$ Hygeia $\quad$ v.16 $\quad$ p. $159-173, \quad 2020$ página 170


Distribuição espacial e aspectos epidemiológicos da leishmaniose tegumentar americana no município de Imbé de Minas, leste do estado de Minas Gerais, Brasil
Rafael Luiz da Silva Neves Andreza do Nascimento Gonçalves Daniel Rodrigues Silva Juscélio Clemente de Abreu Gabriel Menezes Wernersbach Carlos Henrique Wernersbach Guerra

Sandflies. PLoS Neglected Tropical Diseases, 10(3): e0004349, 2016. https://doi.org/10.1371/journal.pntd.0004349

ALVAR, J.; VÉLEZ, I. D.; BERN, C.; HERRERO, M.; DESJEUX, P.; CANO, J.; DEN BOER, M. Leishmaniasis Worldwide and Global Estimates of Its Incidence. PLoS ONE. v. 7, n. 5, e35671, 2012. https://doi.org/10.1371/journal.pone.0035671

BARATA, R. A.; PAZ, G. F.; BASTOS, M. C.; ANDRADE, R. C. O.; BARROS, D. C. M.; SILVA, F. O. L.; MICHALSKY, E. M.; PINHEIRO, C. A.; DIAS, E. S. Phlebotomine sandflies (Diptera: Psychodidae) in Governador Valadares, a transmission area for American tegumentary leishmaniasis in state of Minas Gerais, Brazil. Revista da Sociedade Brasileira de Medicina Tropical, v. 44, n. 2, p. 136-139, 2011. https://doi.org/10.1590/S0037-86822011005000003

BRASIL. Ministério da Saúde. Secretaria de Vigilância em Saúde. Departamento de Vigilância Epidemiológica. Guia de vigilância epidemiológica / Ministério da Saúde, Secretaria de Vigilância em Saúde, Departamento de Vigilância Epidemiológica. $7^{a}$ Edição. Brasília: Ministério da Saúde, 2009.

BRASIL. Ministério da Saúde. Secretaria de Vigilância em Saúde. Manual de Vigilância da Leishmaniose Tegumentar Americana. Edição. Brasília - DF: Ministério da Saúde, 2017.

CÁRDENAS, R; SANDOVAL, C. M; RODRIGUEZ-MORALES, A. J; VIVAS, P. Zoonoses and climate variability: the example of leishmaniasis in southern departments of Colombia. Annals of the New York Academy of Sciences, n. 1149, p. 326-330, 2008. https://doi.org/10.1196/annals.1428.094

CARDOSO, D. T.; SOUZA, D. C.; CASTRO, V. N.; GEIGER, S. M.; BARBOSA, D. S. Identification of priority areas for surveillance of cutaneous leishmaniasis using spatial analysis approaches in Southeastern Brazil. BMC Infectious Diseases, v. 19, p. 1-11, 2019. doi: https://doi.org/10.1186/s12879-019-3940-4

COSTA, J. M. L.; BALBY, I. T. A.; Rocha, E. J. S.; SILVA, A. R.; Rebêlo, J. M. M.; Ferreira, L. A.; GAMA, M. E. A.; BRANCO, M. R. F. C.; BURATTINI, M. N.; SOARES, N. J. Estudo comparativo da leishmaniose tegumentar americana em crianças e adolescentes procedentes das áreas endêmicas de Buriticupu (Maranhão) e Corte de Pedra (Bahia), Brasil. Revista da Sociedade Brasileira de Medicina Tropical, v. 31, p. 279-288, 1998. https://doi.org/10.1590/S0037-86821998000300005

COSTA, S. M. Impactos das mudanças climáticas e ambientais na distribuição espacial de Lutzomyia (Nyssomyia) whitmani (Antunes \& Coutinho, 1939) (Diptera: Psychodidae: Phlebotominae) e no processo de expansão geográfica da Leishmaniose Tegumentar Americana (LTA) no Brasil. Tese (Doutorado). Programa de Pós-Graduação Biodiversidade e Saúde do Instituto Oswaldo Cruz. Rio de Janeiro. 2018.

DAVIES, C. R.; LLANOS-CUENTAS, E. A.; CAMPOS, P.; MONGE, J.; VILLASECA, P.; DYE, C. Cutaneous leishmaniasis in the Peruvian Andes: risk factors identified from a village cohort study. The American Journal of Tropical Medicine and Hygiene, v.56, p. 85-95, 1997. https://doi.org/10.1017/S0950268800057964

DESJEUX, P. Leishmaniasis: current situation and new perspectives. Comparative Immunology, Microbiology \& Infectious Diseases, n. 27, p. 305-318, 2004. https://doi.org/10.1016/i.cimid.2004.03.004

EID, D.; GUZMAN-RIVERO, M.; ROJAS, E.; GOICOLEA, I.; HURTIG, A. K.; ILLANES, D.; SEBASTIAN, M. S. Risk factors for cutaneous leishmaniasis in the rainforest of Bolivia: a cross-sectional study. Tropical Medicine and Health, v. 46, n. 9, 2018. https://doi.org/10.1186/s41182-018-0089-6

ESPINOSA, O. A.; SERRANO, M. G.; CAMARGO, E. P.; TEIXEIRA, M. M. G.; SHAW, J. J. An appraisal of the taxonomy and nomenclature of trypanosomatids presently classified as Leishmania and Endotrypanum. Parasitology, v. 145, n. 4, p. 430-442, 2016. https://doi.org/10.1017/S0031182016002092

FONSECA, E. S.; D'ANDREA, L. A.; TANIGUCHI, H. H.; HIRAMOTO, R. M.; TOLEZANO, J. E.; GUIMARÃES, R. B. Spatial epidemiology of American cutaneous leishmaniasis in a municipality of west São Paulo State, Brazil. Journal of Vector Borne Diseases, v. 51, n. 4, p. 271-275, 2014.

GUERRA O. A. J.; RIBEIRO, J. A. S.; COELHO, L. I. A. R. C.; BARBOSA, M. G. V.; PAES, M. G. Epidemiologia da Leishmaniose Tegumentar na Comunidade São João, Manaus, Amazonas.

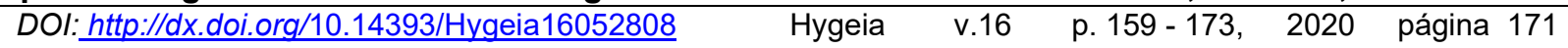


Distribuição espacial e aspectos epidemiológicos da leishmaniose tegumentar americana no município de Imbé de Minas, leste do estado de Minas Gerais, Brasil
Rafael Luiz da Silva Neves Andreza do Nascimento Gonçalves Daniel Rodrigues Silva Juscélio Clemente de Abreu Gabriel Menezes Wernersbach Carlos Henrique Wernersbach Guerra

Cadernos de Saúde Pública, v. 22, n. 11, p. 2319-2327, 2006. https://doi.org/10.1590/S0102$\underline{311 \times 2006001100006}$

GONTIJO, C. M. F.; SILVA, E. S.; FUCCIO, M. B.; SOUSA, M. C. A.; PACHECO, R. S.; DIAS, E. S.; FILHO, J. D. A.; BRAZIL, R. P.; MELO, M. N. Epidemiological studies of an outbreak of cutaneous leishmaniasis in the Rio Jequitinhonha valley, Minas Gerais, Brazil. Acta Tropica, v. 81, n. 2, p. 143-150, 2002. https://doi.org/10.1016/S0001-706X(01)00205-4

GONTIJO, C. M. F; MELO, M. N. Leishmaniose visceral no Brasil: quadro atual, desafios e perspectivas. Revista Brasileira de Epidemiologia, v. 7, p. 338-347, 2004. https://doi.org/10.1590/S1415-790X2004000300011

IBGE - Instituto Brasileiro de Geografia e Estatística. Censo Demográfico 2010. Disponível em: $<$ https://censo2010.ibge.gov.br/> Acessado em 18 de junho de 2018.

IBGE. Instituto Brasileiro de Geografia e Estatística. Imbé de Minas. Disponível em: $<$ https://cidades.ibge.gov.br/brasil/mg/imbe-de-minas/panorama $>$. Acessado em 18 de junho de 2018.

KARAGIANNIS-VOULES, D. A.; SCHOLTE, R. G. C.; GUIMARÃES, L. H.; UTZINGER, J.; VOUNATSOU, P. Bayesian geostatistical modeling of leishmaniasis incidence in Brazil. PLoS Neglected Tropical Disease, v. 7, n. https://doi.org/10.1371/journal.pntd.0002213

KAWA, H.; SABROZA, P. C.; OLIVEIRA, R. M.; BARCELLOS, C. A produção do lugar de transmissão da leishmaniose tegumentar: o caso da localidade Pau-da-fome na cidade do Rio de Janeiro, Brasil. Cadernos de Saúde Pública, v. 26, p. 1495-1507, 2010. https://doi.org/10.1590/S0102-311X2010000800004

LAINSON, R.; SHAW, J. J. Epidemiology and ecology of leishmaniasis in Latin America. Nature, n. 273 , p. 595-600, 1978. https://doi.org/10.1038/273595a0

LOCKARD, R. D.; WILSON, M. E.; RODRÍGUEZ, N. E. Sex-Related Differences in Immune Response and Symptomatic Manifestations to Infection with Leishmania species. Journal of Immunology Research, 4103819, 2019. https://doi.org/10.1155/2019/4103819

MARGONARI, C.; MENEZES, J. A.; ROCHA, M. N.; MAIA, K. N.; OLIVEIRA, M. E.; FONSECA, A. L.; SOUSA, F. F.; FERREIRA, E. C.; MADUREIRA, A. P.; MELO, M. N.; SOARES, R. P. Public Knowledge about and Detection of Canine Visceral Leishmaniasis in Urban Divinópolis. Revista da Sociedade Brasileira de Medicina Tropical, v. 2012: 429586, 2012. https://doi.org/10.1155/2012/429586

MELO, H. A.; ROSSONI, D. F.; TEODORO, U. Spatial distribution of cutaneous leishmaniasis in the state of Paraná, Brazil. PLoS One, v. 12, n. 9, e0185401, 2017. doi: https://doi.org/10.1371/journal.pone.0185401

MENDES, C. S.; COELHO, A. B.; FÉRES, J. G.; SOUZA, E. C.; CUNHA, D. A. Impacto das mudanças climáticas sobre a leishmaniose no Brasil. Ciência e Saúde Coletiva, Rio de Janeiro, v. 21, n. 1, p. 263-272, 2016. https://doi.org/10.1590/1413-81232015211.03992015

MENEZES, J. A.; FERREIRA, C.; ANDRADE-FILHO, J. D.; ALESSANDRA, M. S.; MORAIS, M. H. G.; ROCHA, A. M. S.; MACHADO-COELHO, G. L. L.; LIMA, F. P.; MADUREIRA, A. P.; GARCIA, T. C.; FREITAS, C. R.; SOARES, R. P.; MARGONARI, C. An Integrated Approach Using Spatial Analysis to Study the Risk Factors for Leishmaniasis in Area of Recent Transmission. BioMed Research International, v. 2015: 621854, 2015. https://doi.org/10.1155/2015/621854

NEGRÃO, G. N. Leishmaniose Tegumentar Americana: aspectos geográficos intervenientes na ocorrência da enfermidade no município de Maringá, Paraná. Hygeia - Revista Brasileira de Geografia Médica e da Saúde, v. 5, n. 8, 2009.

OLIART-GUZMÁN, H.; MARTINS, A. C.; MANTOVANI, S. A. S.; BRAÑA, A. M.; DELFINO, B. M.; PEREIRA, T. M.; SANTOS, A. P.; JÚNIOR, J. A. F.; BRANCO, F. L. C. C.; CAMPOS, R. G.; OLIVEIRA, C. S. M.; MUNIZ, P. T.; SILVA-NUNES, M. Epidemiological profile of American cutaneous leishmaniasis in the amazonian frontier: a retrospective study in Assis Brasil, Acre, Brazil. Revista de Patologia Tropical, v. 42, n. 2, p. 187-200, 2013. https://doi.org/10.5216/rpt.v42i2.25522 
Distribuição espacial e aspectos epidemiológicos da leishmaniose tegumentar americana no município de Imbé de Minas, leste do estado de Minas Gerais, Brasil
Rafael Luiz da Silva Neves Andreza do Nascimento Gonçalves Daniel Rodrigues Silva Juscélio Clemente de Abreu Gabriel Menezes Wernersbach Carlos Henrique Wernersbach Guerra

PASSOS, V. M.; FALCÃO, A. L.; MARZOCHI, M. C.; GONTIJO, C. M.; DIAS, E. S.; BARBOSASANTOS, E. G.; GUERRA, H. L.; KATZ, N. Epidemiological aspects of American cutaneous leishmaniasis in a periurban area of the metropolitan region of Belo Horizonte, Minas Gerais, Brazil. Memórias do Instituto Oswaldo Cruz, v. 88, p. 103-110, 1993. https://doi.org/10.1590/S0074$\underline{02761993000100016}$

PINTO, M. O. K. M.; OLIVEIRA, T. M.; AGUIAR, A. N. A.; PINTO, P. E. M.; BARBOSA, D. S.; DINIZ, S. A.; SILVA, M. X. Profile of American tegumentary leishmaniasis in transmission areas in the state of Minas Gerais, Brazil, from 2007 to 2017. BMC Infectious Diseases, v. 20, n. 1: 163, 2020. https://doi.org/10.1186/s12879-020-4860-z

REITHINGER, R.; DUJARDIN, J. C.; LOUZIR, H.; PIRMEZ, C.; ALEXANDER, B.; BROOKER, S. Cutaneous leishmaniasis. The Lancet Infectious Diseases, n. 7, p. 581-596, 2007. https://doi.org/10.1016/S1473-3099(07)70209-8

REY, L. Parasitologia: parasitos e doenças parasitárias do homem nos trópicos ocidentais. $4^{\mathrm{a}}$ edição. Rio de Janeiro: Guanabara Koogan, 2008.

ROSS, R. Further notes on Leishman's bodies. The British Medical Journal, v. 2, n. 2239, p. 1401, 1903. https://doi.org/10.1136/bmj.2.2239.1401

SHAW, J.; PRATLONG, F.; FLOETER-WINTER, L.; ISHIKAWA, E.; EL BAIDOURI, F.; RAVEL, C.; DEDET, J. P. Characterization of Leishmania (Leishmania) waltoni n.sp. (Kinetoplatida: Trypanosomatidae), the parasite responsible for diffuse cutaneous leishmaniasis in the Dominican Republic. The American Journal of Tropical Medicine and Hygiene. n. 93, p. 552-558, 2015. https://doi.org/10.4269/aitmh.14-0774

SOARES, M. E. C.; PEDROSA-SILVA, A. L.; ROCHA, L. S. E.; LIMA, N. L.; VERLI, F. D.; FERREIRA, F. O.; MARINHO, S. A. Epidemiological profile of individuals with American cutaneous leishmaniasis in Jequitinhonha Valley, Brazil. The Eastern Mediterranean Health Journal, v. 19, $\mathrm{n}$. 3, p. 81-88, 2014. https://doi.org/10.26719/2013.19.Supp3.S81

YADÓN, Z. E.; QUIGLEY, M. A.; DAVIES, C. R.; RODRIGUES, L. C.; SEGURA, E. L. Assessment of leishmaniasis notification system in Santiago del Estero, Argentina, 1990 - 1993. The American Journal of Tropical Medicine and Hygiene, v. 65, p. 27-30, 2001. https://doi.org/10.4269/ajtmh.2001.65.27 